Volume 51

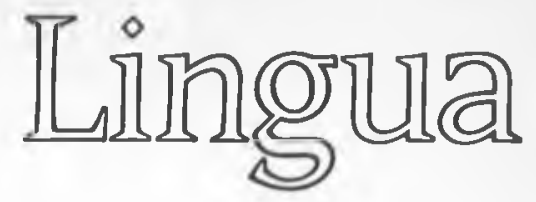

International Review of General

Linguistics

Revue Internationale de

Linguistique Générale

Editors A.J.B.N. Reichling

E.M. Uhlenbeck

W. Sidney Allen

J.G. Kooij

Executive Editor S.C. Dik

North-Holland

Publishing Company

Amsterdam 
All rights reserved. No part of this publication may be reproduced, stored in a retrieval system, or transmitted, in any form or by any means, electronic, mechanical, photocopying, recording or otherwise, without the prior permission of the copyright owner.

Printed in The Netherlands 
Lingua 51 (1980) 17-44

(C) North-Holland Publishing Company

\title{
GRUNDPROBLEME DER KOORDINATION
}

\author{
Bernd WIESE \\ Freie Universität Berlin
}

Received June 1979

\begin{abstract}
Some basic difficulties in the generative description of grammatical coordination are traced back to principal open points in Chomsky's initial model of transformational grammar. The argument is based (i) on an analysis of the role played by internal and external conditions of adequacy for grammars in the conception of syntactic structures, and (ii) on an examination of the relation between syntax and semantics as proposed by Chomsky. The continuation of the problems stated is shown by surveying a number of contributions from later models. As a possible solution a description of coordination in terms of a 'surface syntax for semantics' is envisaged.
\end{abstract}

\section{Einleitung}

Die mit 'Koordination' bezeichneten sprachlichen Erscheinungen haben in der generativen Transformationsgrammatik (TG) ein überaus reges Interesse gefunden. Über 100 Untersuchungen, die sich speziell auf Probleme der Erzeugung koordinierter Einheiten und ihrer Strukturen richten, liegen bis heute vor. Die Gründe für diesen immensen Forschungsaufwand kann man zunächst in den Eigenschaften des sprachlichen Musters Koordination suchen, da dieses erstens zu den Mitteln gehört, die die 'Unendlichkeit' von Sprachen, wie Chomsky sie sieht, garantieren; zweitens seine Untersuchung das von Dik (1968) so genannte Reduktionspostulat - eine Grundidee des Chomskyschen Programms - zu stützen scheint; und drittens Koordination einen sich anbietenden Gegenstand der in der TG angestrebten Universalienforschung bildet. Der vermuteten Allgemeinheit und Einfachheit der Koordinationserscheinungen steht paradoxerweise eine grosse Komplexität der theoretischen Versuche zu ihrer Erfassung und ein immer wiederholter Hinweis der mit diesem Thema beschäftigten Sprachwissenschaftler auf die grosse Vorläufigkeit ihrer Ergebnisse gegenüber. Der nicht versiegen wollende Strom von Papers 
zum Thema weist daher nicht nur auf einen interessanten Gegenstand der Grammatikforschung hin, sondern darf ebenso getrost als Symptom für grundsätzliche Schwierigkeiten gelten, mit den Mitteln der TG den in Rede stehenden Erscheinungen gerecht zu werden.

Angesichts dieser Sachlage scheint es angebracht, die Grundannahmen zu überdenken, die seit Chomskys frühesten Arbeiten in generative Untersuchungen der Koordination eingeflossen sind, und die - trotz aller Aufsplitterungen, Richtungskämpfe und Revisionen - weithin konstant geblieben sind. Diese weiter wirkenden Charakteristika des ersten Modells der TG (Chomsky 1955, 1957, 1975) sind Gegenstand des vorliegenden Aufsatzes. Ihre Untersuchung macht ungelöste Grundprobleme deutlich, die in den späteren Versionen nicht überwunden werden konnten. Dabei richtet sich das Interesse nicht auf technische Fragen oder Einzelheiten der Konjunktionsregeln in verschiedenen Grammatikfragmenten, sondern auf allgemeine Züge der generativen Behandlung des Themas 'Koordination'. Ich erörtere zunächst Chomskys Konzeption syntaktischer Strukturen (§1) und gehe dann den Annahmen zum Verhältnis von Syntax und Semantik nach (\$2). Anschliessend zeige ich die Schwierigkeiten, die diese Konzeption bei der Anwendung des ersten Modells generativer Grammatiken auf die Erscheinungen der Koordination im Deutschen nach sich zog (\$3) und belege schliesslich, wenngleich eher skizzenhaft, die Kontinuität der festgestellten Grundprobleme bei der Untersuchung der Koordination in späteren TG-Modellen ( $\$ 4)$.

\section{Chomskys Konzeption syntaktischer Strukturen}

In seinem frühen Hauptwerk (Chomsky 1955/1975) ${ }^{1}$ stellt Chomsky seine Arbeit ausdrücklich in den Rahmen distributionalistischer Forschungen. In der Zusammenfassung (1955: 717; vgl. 1975: 63) kommentiert er seine Untersuchungen wie folgt:

"Following one of the central tendencies of contemporary descriptive linguistics, we have tried to show that within the framework of 'distributional analysis', we can develop a simple and internally motivated linguistic theory, $[\ldots]$. We conclude that a theory of linguistic form constructed within a 'distributional' basis does delimit an interesting and significant area of linguistic behavior, and that it can be an adequate instrument for the exploration and investigation of this area."

\footnotetext{
${ }^{1}$ Da dieses Werk aufgrund seiner späten Veröffentlichung in praxi nicht die ihm gebührende Aufmerksamkeit gefunden hat, belege ich meine Darstellung mit einigen längeren Zitaten. Herangezogen wird die gedruckte Version von 1975, soweit diese nicht von der ursprünglichen Fassung von 1955 abweicht. Zu den verschiedenen Versionen vgl. die Einleitung zu (Chomsky 1975).
} 
An anderer Stelle erläutert Chomsky sein Verständnis von 'distributional' näher:

"The notions that enter into linguistic theory are those concerned with the physical properties of utterances, the formal arrangements of parts of utterances, conformity of utterance tokens (as determined by the pair test), and finally, formal properties of systems of representation and of grammars. Considerations of other kinds can be admitted if shown to be clear and relevant. We will refer to linguistic analysis carried out in these terms as 'distributional analysis'." (1975:127)

Die Frage nach den Kriterien für die Korrektheit der syntaktischen Strukturen, die eine Grammatik den von ihr erzeugten Sätzen zuschreibt, die hier besonders interessiert, wäre demnach durch den Hinweis auf empirische Untersuchungen zur Distribution von Morphemketten in der jeweils zu untersuchenden Sprache $\mathrm{zu}$ beantworten. Kriterium für Konstituentenstrukturen wäre also, dass die in den Strukturbäumen gekennzeichneten Konstituenten tatsächlich distributionelle, ermittelbare Einheiten wären. Hieraus könnte man eine deutliche Entscheidung der Frage entnehmen, ob syntaktische Strukturen einen empirischen Status hätten, wie sie im amerikanischen Strukturalismus vor Chomsky diskutiert wurde. (Vgl. z.B. Hockett 1948.) Dass die Entscheidung in Wahrheit nicht so eindeutig ausfiel, wird sich im folgenden zeigen. Zum einen wurde der Bezug auf beobachtbare Daten von Chomsky durch den Bezug auf "the speaker's 'linguistic intuition"” (Chomsky 1975: 101) ersetzt. Zum zweiten erhielt aus der Erläuterung von 'distributional' gerade der Passus "notions $[\ldots]$ concerned with [...] formal properties [...] of grammars" grösste Bedeutung.

Entscheidend war die Einführung eines Einfachheitskriteriums für Grammatiken, das über die übliche wissenschaftstheoretische Forderung an Theorien hinausgehen sollte. Grob gesagt, schien Chomsky folgendes zu intendieren. Es soll eine allgemeine Grammatiktheorie ("general theory of linguistic structure", Chomsky 1975: 77) aufgestellt werden, die festlegt, welche formalen Eigenschaften Grammatiken besitzen. Unter verschiedenen Grammatiken, die für eine Sprache zur Auswahl gestellt werden, soll diejenige als adäquater gelten, die in dem Sinne einfacher ist, dass sie sich der allgemeinen Definition von Grammatik derart unterordnet, dass auch für alle anderen Sprachen möglichst einfache Grammatiken gefunden werden können, die der Grammatik-Definition entsprechen. 'Einfachheit' könnte nun für Grammatiken definiert werden, ohne sich auf eine methodologische Forderung zu reduzieren: Insofern die allgemeine 
Definition von Grammatik "in a sense" als "definition of "language"” (Chomsky 1975: 81) angesehen würde, sollte die Frage, wie das Einfachheitskriterium auszusehen hätte, zu einem empirischen Problem werden, das gerade in der allgemeinen Formulierung dessen läge, was eine Grammatik sei. ${ }^{2}$ Die "necessity to meet the external conditions of adequacy" (Chomsky 1975: 81), d.h. die empirische Grundlage der Grammatik, geriet gegenüber solchen Erwägungen in den Hintergrund.

Die Folgen für den Aufbau von Konstituentenstrukturen bestanden, allgemein gesprochen, vor allem darin, dass die behauptete Empirizität des Einfachheitskriteriums es erlaubte, Einfachheit nicht mehr als Auswahlkriterium zwischen im übrigen adäquat erscheinenden Analysen zu verwenden, sondern den Aufbau syntaktischer Strukturen unmittelbar durch Erwägungen über die Form der Grammatik, statt durch Erwägungen über den zu beschreibenden Gegenstand zu steuern. Damit war die Entscheidung für einen empirischen Status syntaktischer Strukturen bei Chomsky von Anfang an in starkem Masse zurückgenommen.

Zur Begründung syntaktischer Strukturen stellt Chomsky (1975: 225), nachdem er gewisse andere von ihm verwendete Kriterien bei der Ansetzung von Konstituentenstrukturen benannt hat, auf die wir sofort kommen werden, fest:

"The particular criteria of analysis that we set up have only the function of collecting under one heading certain related ways of simplifying the grammar. The ultimate criterion is total systematic simplicity, and to this there are no exceptions."

Der übliche Sinn von Beschreibung und die damit an Konstituentenstrukturen als 'structural descriptions' gestellten Forderungen kommen nur durch die Hintertür teilweise wieder hinein. Die 'tatsächlichen' Strukturen der durch die Grammatik aufzuzählenden Sätze liefern in gewisser Weise Kriterien, weil sich die Aufzählung vereinfacht, wenn man sie mit heranzieht $(1975: 210)$ :

"To the question: what is ultimately involved in the proper choice of $P$ [i.e. einer 'Ebene' der Konstituentenstruktur (level of phrase structure) - B.W.], for a given language, we have given the general answer: the total simplicity of the grammar for which $P$ provides the underlying phrase structure. More specifically, the more features of the language can be stated in terms of the elements of $P$, the simpler will be the grammar which $P$ underlies, since then these features will not have to be independently stated. Thus each such feature

${ }^{2} \mathrm{Zu}$ einer gründlichen Kritik der hier involvierten Idee einer 'Sprachtheorie als Grammatiktheorie' vgl. Lieb (1974). Entscheidende Einwände gegen den Versuch, den Einfachheitsbegriff sprachpsychologisch zu validieren gibt z.B. Watt (1974). 
can be taken in a loose sense as a criterion for $P$. This is actually a loose sense, because the only ultimate criterion for $\boldsymbol{P}$ is the total simplicity of the grammar. Simplicity is a measure for a whole system. [...] The following features appear to be criteria for $P$ in English, in this loose sense. [...]

1. The rule for conjunction

2. Intrusion of parenthetical expressions

3. Ability to enter into transformations

4. Certain intonational features."

Im vorliegenden Zusammenhang interessiert das erste dieser Kriterien. Es wird von Chomsky als das wichtigste der "criteria in a loose sense" angesehen. Das gleiche Kriterium wird auch in Chomsky (1957: 36) aufgestellt:

"In fact, the possibility of conjunction offers one of the best criteria for the initial determination of phrase structure."

und in Chomsky (1975) in verschiedener Weise reformuliert. Z.B. (224):

"The rule giving the distribution of 'and' can be used as a criterion for constituent analysis [...]."

Dieses 'Konjunktionskriterium' bleibt dem Einfachheitskriterium untergeordnet. Seine Beachtung soll nur zur Vereinfachung beitragen, da man, statt alle Sätze in denen und-Konstruktionen vorkommen je für sich zu erzeugen, diese Sätze aus ohnehin bereits erzeugten Strukturen, die nicht konjugierten Sätzen entsprechen, gewinnen kann, wenn man eine geeignete Regel hat. Nun entspräche es durchaus der proklamierten distributionalistischen Grundlage, derartige Beziehungen zwischen Sätzen als heuristische Kriterien zur Bestimmung der Satzstrukturen zu verwenden. Man würde demnach annehmen, dass Teile von Sätzen, die sich durch geeignete Transformationen konjugieren lassen, im allgemeinen als Konstituenten betrachtet werden sollten. Da es Chomsky aber nicht um die Aufdeckung von Strukturen, sondern um eine dem Einfachheitskriterium genügende Ansetzung von Strukturen geht, ist ein transformationelles Kriterium nur dann von Interesse, wenn die Grammatik tatsächlich eine Regel enthält, die die transformationelle Beziehung wiedergibt. Mit anderen Worten, eine Vereinfachung der Grammatik tritt nur ein, wenn in die Grammatik eine Konjunktionsregel auch wirklich aufgenommen wird. Kommt eine solche Regel gar nicht vor, so bildet sie auch kein Kriterium unter dem Gesichtspunkt der Einfachheit. Nun ist aber die Konjunktionsbeziehung für Chomsky in der Tat das wichtigste Kriterium für die Aufstellung der Konstituentenstrukturen in den Ersetzungssystemen, die er zur 
Rekonstruktion strukturalistischer Konstituentenanalysen vorschlägt. Er muss daher am Ende seiner Diskussion dieses Grammatiktyps (Chomsky 1975: 290) feststellen: "Hence if the grammar cannot incorporate this rule, the proposed approach to a demonstration of validity is undermined [...]".

Genau dies ist aber der Fall, wenn die Grammatik nur aus Ersetzungsregeln besteht. Chomsky gelangt so zur Einführung der Transformationsregeln, für die die Konjunktionsregel ein prominentes Beispiel bildet. Die transformationelle Ableitbarkeit wird selbst als Bestandteil der Struktur von Sätzen aufgefasst, die somit (neben einer Konstituentenstruktur) eine Transformationsstruktur besitzen. Die dahinter liegende Idee ist das eingangs erwähnte Reduktionspostulat: Einfache Sätze ("basic sentences (simple, declarative, active, with no complex verb or noun phrases)", Chomsky 1957: 106f) werden über die ihnen entsprechenden Ketten der Phrasenstrukturebene transformationell mit komplexen Sätzen verbunden. Das Reduktionspostulat führt aber, insbesondere bei koordinierten Einheiten, zu mehrfacher Ableitbarkeit und somit zu einer Komplizierung der Grammatik.

Chomskys Rechtfertigung besteht in einem Rückgriff auf die 'Sprecherintuitionen', die, wie erwähnt, die empirische Basis vertreten sollen. Sie liefern 'externe' Adäquatheitskriterien, die den 'internen' Einfachheitsanforderungen entgegenstehen können. Die mehrfache Ableitbarkeit soll dann gegeben sein, wenn der abgeleitete Satz nach Sprecherintuitionen im selben Masse 'ambig' ist (Chomsky 1957: 86):

"We can test the adequacy of a given grammar by asking whether or not each case of constructional homonymity is a real case of ambiguity and each case of the proper kind of ambiguity is actually a case of constructional homonymity."

Wann ist nun ein Satz 'ambig', und was ist es, dass ihn 'ambig' macht? Ambig oder mehrdeutig, so läge es nahe anzunehmen, ist ein Satz, wenn ihm mehrere Bedeutungen zugeschrieben werden können. Mehrdeutigkeit wäre daher ein semantischer Begriff und 'Sprecherintuition' stünde für Kenntnis der Bedeutungsseite der betreffenden Sprache. Dies würde zunächst noch keine Probleme aufwerfen, wäre nicht Chomsky ein pointierter Vertreter der Auffassung, dass jegliche semantischen Erwägungen bei syntaktischen Untersuchungen nicht nur nichts zu suchen haben, sondern in gar keiner Weise nutzbar zu machen seien. Diese Konzeption Chomskys von einer 'autonomen Syntax' soll daher im folgenden Abschnitt untersucht werden, bevor wir dem Ambiguitätskriterium weiter nachgehen wollen. 


\section{Chomskys Konzeption einer 'autonomen Syntax'}

Nach traditioneller Auffassung ist eine Syntax derjenige Teil einer Grammatik, "was schon das Wort besagt, dessen Aufgabe es ist, darzulegen, wie die einzelnen Wörter zum Zwecke der Mitteilung zusammengeordnet werden" (Paul, 1959: 3). Wenngleich unscharf, umreisst diese Formulierung das alte Programm einer semantikfundierenden Syntax. In Syntactic Structures widmet Chomsky dem Verhältnis von Syntax und Semantik ein eigenes Kapitel. Ganz im Sinne der alten Ziele der Syntaxforschung schreibt er dort (1957: 102):

"We should like the syntactic framework of the language that is isolated and exhibited by the grammar to be able to support semantic description, and we shall naturally rate more highly a theory of formal structure that leads to grammars that meet this requirement more fully."

Hier wird der semantikfundierende Charakter der Syntax angesprochen ("... support semantic description ...") und darüberhinaus als ein Bewertungskriterium für Grammatiken in Anspruch genommen ("... rate more highly ..."). ${ }^{3}$ Weiterhin nimmt Chomsky auch an, dass die von ihm behandelten syntaktischen Strukturen derartigen Forderungen genügen (1957: 102):

"Phrase structure and transformational structure appear to provide the major syntactic devices available in language for organization and expression of content."

Lässt man einige Unklarheiten ausser acht, die sich aus Chomskys damaliger Annahme einer 'Gebrauchstheorie der Bedeutung' ${ }^{4}$ ergeben, so kann man aus den Ausführungen des zugrundegelegten Kapitels in etwa herauslesen, was dies im einzelnen besagen soll. Chomsky zielt darauf ab, zu zeigen, dass transformationell erzeugte Sätze in Hinblick auf die den

\footnotetext{
${ }^{3}$ Vgl. auch die entschiedenere Formulierung einer Vorlesungspassage von 1964, in der Chomsky ausdrücklich auf Syntactic Structures verweist: "And it would be absurd to develop general syntactic theory without assigning an absolutely crucial role to semantic considerations, since obviously the necessity to support semantic interpretation is one of the primary requirements that the structures generated by the syntactic component of a grammar must meet" (Chomsky 1966: 20, Fn.).

${ }^{4}$ In Chomsky/Parret (1974: 37) schreibt Chomsky: "In Syntactic Structures I assumed a "use theory' of meaning under the influence largely of Wittgenstein and Oxford philosophy". Sie deutet sich dort in Formulierungen wie z.B. "the correlations between the form and use of language" (1957: 102) an. Die Vorstellung von 'Gebrauchstheorie' oder 'Bedeutung als Gebrauch' bleibt in Syntactic Structures jedoch dunkel und wird durch Erläuterungen wie "the actual use of language, i.e. [...] the process of understanding language" (103) nicht gerade aufgehellt.
} 
Sätzen zugrundeliegenden Strukturen verstanden werden, die ihrerseits durch Ersetzungsregeln erzeugt werden. Die Kernsätze, d.h., die einfachsten Sätze, die am unmittelbarsten den vom Ersetzungsteil der Grammatik erzeugten Strukturen entsprächen, können nämlich nach Chomsky als "the 'elementary content elements' out of which [a] sentence is constructed" (Chomsky 1957: 108) aufgefasst werden. Demnach ergibt sich für Chomsky (92):

"The general problem of analyzing the process of 'understanding' is thus reduced, in a sense, to the problem of explaining how kernel sentences are understood, these being considered the basic 'content elements' from which the usual, more complex sentences of real life are formed by transformational development".

Natürlich muss man darüberhinaus noch wissen, welchen transformationellen Veränderungen die Kernsätze unterzogen worden sind, um einen vorliegenden Satz zu bilden.

Chomskys Syntax soll also die Aufgabe, eine Grundlage für die Semantik zu liefern, lösen, indem Zeichenketten, die Sätzen transformationell zugrunde liegen, Bedeutungen zugeschrieben werden und Bedeutungsmodifikationen, die sich aus Transformationen ergeben, berücksichtigt werden. (Vg1. die Ausarbeitung von Katz und Fodor 1963.)

Wichtiger als die Einzelheiten dieser Konzeption, die an Chomskys erstes Grammatikmodell gebunden bleiben, sind jedoch diejenigen Annahmen, die dieses runde Verhältnis von Syntax und Semantik trüben. Es gibt nämlich Feststellungen, die das obige Gesamtbild nicht nur beeinträchtigen, sondern ihm entgegenstehen. Diese betreffen den Kern dessen, was Chomskys 'Autonomie der Syntax' ausmacht. Obwohl nämlich die Semantik nicht einfach aus der Grammatik zu verbannen sei, so sei doch die Beziehung zwischen Syntax und Semantik durch ihre Inexaktheit gekennzeichnet und liefere deshalb keine Gesichtspunkte für die Syntaxforschung:

\footnotetext{
"It seems clear, then, that undeniable, though only imperfect correspondences hold between formal and semantic features in language. The fact that the correspondences are so inexact suggests that meaning will be relatively useless as a basis for grammatical description." (1957: 101, meine Hervorhebung)
}

Und ähnlich (1957: 108):

"It is questionable that the grammatical devices available in language are used consistently enough so that meaning can be assigned to them directly." 
Mit diesen Erwägungen sind zwei auseinanderzuhaltende Punkte angesprochen, zum einen die Frage, wie Syntax und Semantik systematisch aufeinander zu beziehen sind, zum anderen die Frage, welche Rolle semantischen Erwägungen methodologisch in der Syntaxforschung zuzuschreiben ist.

Die systematische Frage beantwortet Chomsky mit der Zurückweisung 'direkter Bedeutungszuordnungen', für die etwa die Zuordnung einer 'strukturellen Bedeutung Agent-Aktion' zur grammatischen 'SubjektVerb' Relation ein Beispiel bilden würde (vgl. 1957: 94). Nun folgt aber aus der Tatsache, dass eine 'direkte' Zuordnung, insbesondere eine Eins-zu-eins-Zuordnung von Bedeutungen zu Formelementen, nicht möglich ist, keineswegs schon eine Unexaktheit oder Unvollständigkeit der Entsprechungsbeziehung von Form und Bedeutung. Die Zuordnung muss eben eine andere als eine in Chomskys Sinn 'direkte' sein, und tatsächlich liefert er selbst ja einen Vorschlag zu einer indirekten Zuordnung in Form der Auffassung, die Kernsätze entsprächen den 'basic content elements'.

Auf der methodologischen Seite führt die Einsicht des amerikanischen Strukturalismus, dass die syntaktischen Beschreibungen nicht durch semantische Erwägungen zu begründen seien, bei Chomsky zu einem Missverständnis hinsichtlich der Rolle semantischer Erwägungen für die Syntax, das aus der Harrisschen Tradition herrührt. Lyons spiegelt dieses Missverständnis wieder, wenn er Harris' Schule attestiert (1968: 212):

\footnotetext{
"Wells and Harris formulated the principles of constituent analysis in greater detail and replaced Bloomfield's somewhat vague reference to 'taking account of meanings' with explicitly distributional criteria."
}

In Wahrheit können distributionelle Kriterien keineswegs als 'Ersatz' für Bloomfields Bezugnahme auf Bedeutungen dienen. Wenn Bloomfield schreibt, dass eine "proper analysis [of constituents]" eine solche sei, "which takes account of meanings" (Bloomfield 1965: 161) - und damit seine Interpreten Lügen straft, die ihm eine "Verdrängung von 'meaning' aus der exakten Sprachbeschreibung" (Helbig 1971: 76) anlasten -, so spricht er nicht darüber, worauf man sich bei der systematischen Begründung syntaktischer Strukturen stützen darf. Bloomfield nahm vielmehr auf Bedeutung Bezug, um zu überprüfen, ob vorgeschlagene syntaktische Strukturen in dem Sinne adäquat seien, dass sie zur Erklärung der Vermittlung von Bedeutungen durch sprachliche Äusserungen herangezogen werden können. 
Es stellt nun eine Vermengung der systematischen und der methodologischen Frage dar, wenn Chomsky im Unterschied zu Bloomfield die Ansicht vertritt, dass mit der systematischen Vorordnung der Syntax vor die Semantik und der Notwendigkeit, syntaktische Begriffe formal zu konstruieren, eine heuristische Verwendung semantischer Erwägungen sowie eine Überprüfung syntaktischer Strukturen in Hinblick auf semantische Erwägungen nicht zu vereinbaren sei. Genau genommen, behauptet Chomsky nicht, dass derartige Erwägungen nicht zulässig seien, sondern vielmehr gar nicht denkbar seien! Es sei zwar nicht möglich, zu beweisen, dass es ein derartiges Vorgehen nicht geben könne: jedoch liessen sich alle in dieser Richtung gebrachten Vorschläge widerlegen. Man könne daher feststellen: "There is, however, little evidence that "intuition about meaning' is at all useful in the actual investigation of linguistic form" (1957: 94). Gewicht wird nicht nur auf die heuristische Nutzlosigkeit semantischer Überlegungen, sondern vor allem auf die Unmöglichkeit einer Bewertung syntaktischer Theorien in Hinblick auf die Semantik gelegt (1957: 93):

"I am not acquainted with [...] any specific and rigorous proposal for the use of semantic information in [...] evaluating grammars."

Das Fehlen eines solchen Vorschlags ist nach Chomsky nicht ein bedauerlicher Zustand, dem abgeholfen werden sollte, sondern spricht für die Unmöglichkeit einer solchen Bemühung.

Damit stellt sich aber Chomsky in Widerspruch zu seinen Forderungen, die durch das auf S. 23 angeführte Zitat belegt wurden, in dem ausdrücklich von einer Bewertung von Grammatiken in Hinblick auf ihre Leistung zur Stützung der semantischen Beschreibung die Rede ist. Man mag nun annehmen, dass nach Chomskys Auffassung diese Betrachtung unter semantischem Blickwinkel der Syntax in irgendeinem Sinne 'äusserlich' bleibt. Kommen wir jedoch auf das oben diskutierte Ambiguitätskriterium zurück! Mit diesem Kriterium wird der Berücksichtigung von Mehrdeutigkeit eine höchst wichtige Rolle für die syntaktischen Strukturen selber zugewiesen. Soll nun ein offener Widerspruch zum Gedanken der Autonomie der Syntax vermieden werden, so muss der Ambiguitätsbegriff als nicht-semantischer aufgefasst werden. Dies geschieht mit der Annahme, dass die Sprecherintuitionen, die in dieses Kriterium einfliessen, nicht 'intuition about meaning', sondern "intuition about linguistic form" (1957: 93f) seien. Beides werde verwechselt, wenn gefragt würde: "How can you construct a grammar with no appeal to meaning?" (1957: 93). 
Dieser Unterscheidung kann jedoch im Hinblick auf den Begriff 'Ambiguität' nur schwer ein klarer Sinn gegeben werden, zumal Chomsky die der Konstruktionshomonymität korrespondierende Ambiguität ausdrücklich damit in Verbindung bringt, dass Sätze "are understood in more than one way" (1957: 92). Das Verhältnis des Ambiguitätskriteriums zum 'ultimate criterion' der 'total systematic simplicity' bleibt undeutlich. Da er jedoch ersteres unter die "rough criteria" (1957: 102) rechnet, scheint es letztlich hinter dem Einfachheitskriterium zurückstehen zu müssen.

Abschliessend lässt sich Chomskys Auffassung vom Verhältnis zwischen Syntax und Semantik wie folgt kennzeichnen. Der Wunsch nach grösster Strenge hinsichtlich der systematischen Unabhängigkeit der Syntax von der Semantik führt zu einer doppelten Fehlkonzeption. Erstens wird der unproblematische und praktisch unerlässliche heuristische Rückgriff auf die Semantik beim Aufbau syntaktischer Strukturen verurteilt. Zweitens stellt sich Chomsky mit der Zurückweisung semantischer Bewertungskriterien für Syntaxen nicht nur in Widerspruch zu von ihm selbst erhobenen Forderungen, sondern schliesst, jedenfalls theoretisch eine Hauptquelle für Erwägungen aus, die überhaupt erst eine Entscheidung darüber möglich machen, ob eine Syntax die ihr traditionell zugeschriebene Aufgabe erfüllt, die ähnlich wie oben Paul etwa Bloomfield als die Kennzeichnung der "meaningful arrangements of forms in a language" (1965: 163) ansieht.

\section{Koordination im ersten Modell der TG}

Koordinierte Einheiten werden in Syntactic Structures gemäss dem Reduktionspostulat transformationell auf einfache Sätze zurückgeführt. Hierzu führt Chomsky die folgende, Regel ein, die, wie erwähnt, durch die in Anspruch genommene Vereinfachung der Grammatik und ihre Begründungsfunktion für Konstituentenanalysen gerechtfertigt wird (1957: 36):

"If $S_{1}$ and $S_{2}$ are grammatical sentences, and $S_{1}$ differs from $S_{2}$ only in that $X$ appears in $S_{1}$ where $Y$ appears in $S_{2}$ (i.e., $S_{1}=\ldots X .$. and $S_{2}=\ldots Y$. ), and $X$ and $Y$ are constituents of the same type in $S_{1}$ and $S_{2}$, respectively, then $S_{3}$ is a sentence, where $S_{3}$ is the result of replacing $X$ by $X+$ and $+Y$ in $S_{1}$ (i.e., $S_{3}=\ldots X+$ and $+Y$. .)."

Die Regel legt fest:

(i) Die zu koordinierenden Teile der Sätze müssen Konstituenten dieser Sätze sein. 
(ii) Sie müssen Konstituenten der gleichen Kategorie sein.

(iii) Sie müssen in den Ausgangssätzen an gleicher Stelle auftreten.

(iv) Die Ausgangssätze müssen bis auf diese Stelle übereinstimmen.

"Nicht hinreichend geklärt" ist, wie Braun (1967: 75, Fn. 1) bemerkt, die Frage welche Konstituentenstrukturen den durch die Transformation abgeleiteten Ketten zugeordnet werden sollen.

Wichtiger ist aber im vorliegenden Zusammenhang die Frage nach dem Status von (i) bis (iv). Nach Braun (1967: 73) sind Beispiele wie (1) geeignet (i) oder auch Chomskys Diktum

(1) das alte und das graue haus

(v) zu widerlegen (Chomsky 1957: 36, Fn.):

(v) "To form fully grammatical sentences by conjunction, it is necessary to conjoin single constituents."

Demgegenüber will Chomsky aber Koordinierbarkeit als Kriterium für die Konstituentenbildung verwenden. Danach könnte es zu (v) keine Gegenbeispiele geben, weil die Konstituentenstrukturen immer so angesetzt werden müssten, dass (v) zutrifft. Jedoch sieht er das Kriterium ja nicht als absolut an, sondern nur als ein 'loose criterion'. Es erweist sich hier bereits, dass die Interpretierbarkeit der Chomskyschen Regel durch die oben geschilderte Unklarheit der Gegenüberstellung 'interner' und 'externer' Adäquatheitskriterien wesentlich beeinträchtigt wird.

Dass Braun (1) als Gegenbeispiel zu (v) vorlegt, weist sofort auf die zweite oben diskutierte Schwierigkeit hin, die Unklarheit des Verhältnisses von Syntax und Semantik. Der Gesichtspunkt des 'supporting character' syntaktischer Strukturen für die Semantik scheint sich bei Braun durchzusetzen, wenn er ohne nähere Begründung annimmt, dass die verknüpften Teilketten (nach Braun das alte und das graue) keine Konstituenten sein können.

Würde man Chomskys Forderung nach 'asemantischem' Aufbau von Konstituentenstrukturen konsequent verfolgen, so müsste allerdings (1) als einer 'uneingeschränkt grammatischen' Einheit die in Abb. 1 dargestellte Struktur zugewiesen werden, die denn auch in Gleitman (1965), der umfassendsten Arbeit zum Thema Koordination im ersten TG-Modell, immerhin erwogen wird (273, Fn. 19). 


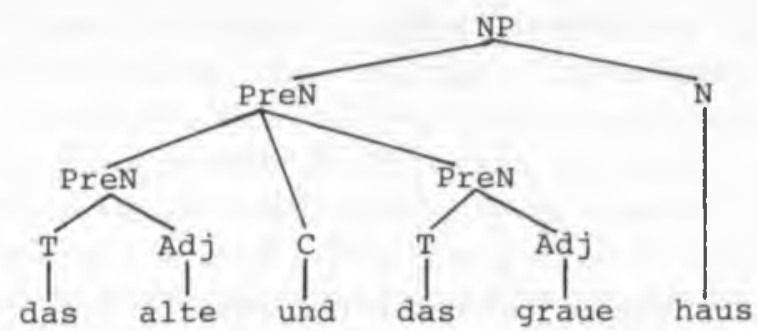

Abb. 1. Koordination von 'Pränominalen' (T: Artikel; Adj: Adjektiv; N: Nomen; PreN: Pränominal; NP: Nominalphrase; C: Konjunktion).

Die vielfältigen Diskussionen um (v), die die ganze spätere TG-Literatur zur Koordination durchziehen, stehen damit von Anfang an im Zeichen einer grundlegenden Unklarheit: Wenn (v) ein Prinzip für die Ansetzung von Konstituentenstrukturen ist, ist die Frage, ob Konjunkte einer Koordination immer Konstituenten sind, durch die Konzeption vorentschieden und kann sich gar nicht mehr als empirisches Problem stellen. Soll (v) aber als empirische Hypothese verstanden werden, so muss die Korrektheit von Konstituentenstrukturen durch andere Prinzipien entschieden werden. Diese liegen dann unausgesprochen in Anforderungen der semantischen Plausibilität der anzunehmenden Konstituentenstrukturen, die als 'Sprecherintuitionen' einbezogen werden.

Die gleiche Unklarheit zeigt sich bei dem Problem, dass es zu 'Überstrukturierungen' kommt, wenn als 'Input' der Konjunktionstransformation immer zwei Ketten anzunehmen sind. Man erhält mit der Chomskyschen Regel für Einheiten wie hans und fritz und karl Strukturen gemäss Abb. 2, nicht aber gemäss Abb. 3 .
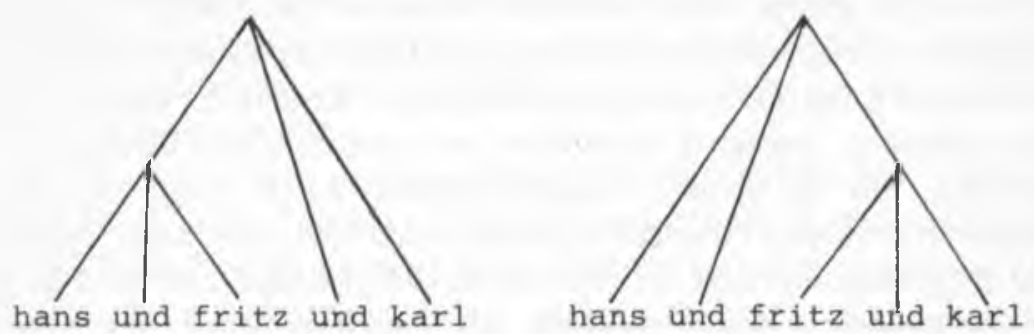

Abb. 2. Überstrukturierung bei Koordination.

Zur Erzeugung von Strukturen wie in Abb. 3 wird in Gleitman (1965) angenommen, dass die Konjunktionstransformation über einer nicht 


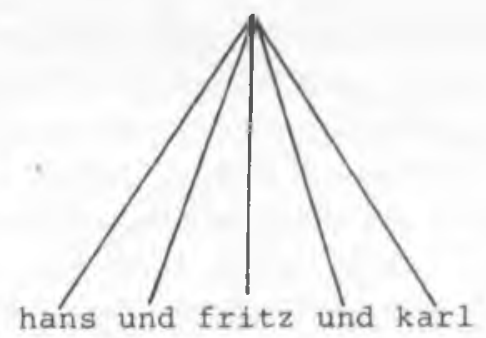

Abb. 3. Mehrfache Koordination.

generell festgelegten Anzahl von Ketten operiert, d.h. die Konjunktionstransformation wird als sogenanntes Regelschema formuliert. Dies wird wie folgt begründet (273, Fn. 20):

"If conjunction is limited to two strings, a complex phrase structure must be built up to describe sentences with more than one conjoining particle. Not only is this picture of phrase structure counter-intuitive, but it creates two problems of description: (1) the rule for the deletion of the conjoining particle (comma intonation) becomes difficult, and (2) the distinction between coordinate and subordinate conjunction becomes obscure."

Man findet wieder die vermischte Anführung von Kriterien für Strukturansetzungen: Intuition ("counter-intuitive"), Einfachheit ("the rule [...] becomes difficult") und Adäquatheit für die Semantik oder Adäquatheit für die Beschreibung syntaktischer Kategorisierungen, die als gültig angenommen werden ("the distinction [...] becomes obscure"). Wichtig ist, dass die Einführung von Regelschemata deswegen nötig wird, weil das Format der verwendeten Regeln festlegt, welche syntaktischen Strukturen überhaupt möglich sind und dabei solche ausschliesst, die aus 'externen' Gründen als wünschenswert erscheinen. Durch die Aufnahme eines neuen Regeltyps wird an dieser Situation aber nichts Grundsätzliches geändert. Nach wie vor richtet sich die Form der Sätzen zugeordneten syntaktischen Strukturen nach den verfügbaren Regeln. Es wird nicht der Schritt gemacht, zunächst die Form der syntaktischen Strukturen zu bestimmen, um diese dann möglicherweise durch geeignete Regeln spezifizieren zu lassen. Gegenüber dieser Grundfrage ist es dann schon von geringerer Bedeutung, dass, wie Thümmel (1970) bemerkt, die Verwendung von Regelschemata nicht geeignet ist, die Zuordnung der falschen Strukturen zu verhindern, da eine wiederholte Anwendung von Regelschemata ebenfalls Strukturen wie in Abb. 2 liefert. $^{5}$

${ }^{5}$ Thümmel entschliesst sich deshalb, aus der Not eine Tugend zu machen, und die Überstrukturierung (bei einer rechts-rekursiven Einbettung koordinierter Sätze) "als 
Der Widerstreit zwischen 'externen', empirischen Forderungen an Konstituentenstrukturen und 'internen', konzeptuellen Forderungen zeigt sich ebenfalls bei dem Teil von Chomskys Konjunktionsregel, der besagt, dass die Konjunkte Konstituenten der gleichen Kategorie sein müssten. Hierin könnte wieder eine empirische Annahme gesehen werden; andererseits wird z.B. auch in Gleitmann (1965) so verfahren, dass der Regelapparat so eingerichtet wird, dass die Forderung erfüllt werden muss. So stellt Gleitmann fest, dass Sätze wie

(2) he took john home and mary to the station

nicht ohne weiteres als Koordination zweier Sätze beschrieben werden können, da die Zuordnung von mary to the station zu S(atz) die Ableitung anderer, ungrammatischer Sätze gestatten würde. Dennoch kann die Forderung der Kategoriengleichheit für Konjunkte erfüllt werden, indem die Konjunktionstransformation die Sätze he took john home und he took mary to the station in einen Satz umformt, der anschliessend durch eine Tilgungsformation $\mathrm{zu}(2)$ verwandelt wird.

Für andere Gegenbeispiele gegen die Annahme der Kategoriengleichheit der Konjunkte, wie sie immer wieder angeführt werden, steht das folgende aus Weydt (1976):

(3) une fille charmante et dont j'ai oublié le nom

Auch im Deutschen sind Nominale und mit Fragepronomina eingeleitete Nebensätze als Konjunkte einer Koordination möglich. Wegen des ungeklärten Status von Chomskys Annahme kann jedoch nie ausgeschlossen werden, dass die Kategoriengleichheit durch Ansetzung einer geeigneten Kategorie erreicht wird, da die syntaktischen Kategorien ja ihrerseits wiederum nur hinsichtlich ihrer Rolle in der Grammatik gekennzeichnet werden.

Die Ausscheidung semantischer Adäquatheitskriterien erweist sich einerseits als Scheinmanöver, indem diese durch die Berücksichtigung von 'Sprecherintuitionen' wieder hineingenommen werden. Andererseits stellt sie eine Immunisierungsstrategie gegen empirische Kriterien für die Korrektheit dar, weil der Begriff der 'intuition about linguistic form' für

abstrakte Abbildung des Nacheinanders der Sätze bei der Sprachanwendung [zu] interpretieren" (1970: 153). Ein gutes Beispiel dafür, wie die Existenz bestimmter Eigenschaften des Regelapparats sachliche Annahmen motiviert statt umgekehrt. 
sich und in seiner Gegenüberstellung zu den 'internen Adäquatheitskriterien' zu unklar bleibt, als dass er eine empirische Überprüfung der Grammatik erlauben würde.

Das Problem der Beliebigkeit der Kategorienansetzung, die den empirischen Charakter der These über die Kategoriengleichheit in einer generativen Grammatik in Frage stellt, geht jedoch noch über die Frage des Verhältnisses interner und externer Adäquatheitsbedingungen hinaus. Es mag daher angebracht sein, zum Schluss dieses Abschnitts die folgenden Bemerkungen anzuschliessen.

Hawkey (1970: 206f) stellt den unbefriedigenden Charakter der syntaktischen Kategorien einer generativen Grammatik deutlich heraus:

"If we ask what a $X$ is, or more precisely, what properties characterize members of the category $X$, the grammar is silent, and dumbly continues to point out the members. [...] It is only in terms of the grammar as a whole, if at all, that we are able to gain some information about the properties which characterize members of categories, and this information can be gained only indirectly and by inference."

Die Grundlage hierfür liegt, wie Hawkey im selben Aufsatz (1970: 200) bemerkt, in der "curious, but significant, identification" der Begriffe "device, which produces ..." und "theory which gives an adequate account of ...". Die Tatsache, dass generative Grammatiken keine Theorien sind und daher über die als Gegenstände in Anspruch genommenen Sprachen keine Aussagen machen, ist zuerst von Lieb herausgestellt worden (vgl. die Zusammenfassung der betreffenden Ergebnisse in Lieb 1974: \$1). Dass generative Grammatiken nicht als Theorien angesehen werden können, wird auch von Autoren zugegeben, die darin keinen Mangel sehen. Etwa schreibt Babitch (1977: 339):

"Transformational grammar claims to be an axiomatization of language. Its theorems neither refer to, nor are pictures of anything. The grammar does not speak about, but shows the structure of a given language. This is why a referential metagrammar is needed."

und bewertet diese Situation unmittelbar anschliessend wie folgt:

"This high degree of abstraction is excellent from the point of view of theoretical consideration."

Abgesehen davon, dass eine Begründung für diese Ansicht nicht gegeben wird, bleibt festzustellen, dass die benötigten Metagrammatiken nicht existieren. 


\section{Koordination in späteren Modellen der TG}

War bei Chomsky $(1955,1957)$ die Einfachheit der Grammatik für den Aufbau syntaktischer Strukturen entscheidend gewesen, so wurden im zweiten Modell der generativen Grammatik, das seine Formulierung in Chomsky (1965) fand, semantische Erwägungen, insbesondere anknüpfend an Chomskys oben erörtertes Ambiguitätskriterium, stärker in den Vordergrund gestellt. Bei dieser Betrachtungsweise ergaben sich bei der Behandlung der Koordination Probleme, die eines der Grundprinzipien des ersten Modells, das Reduktionspostulat, erschütterten. Als relevant wurden Beispiele von zweierlei Art angesehen. Zum einen fanden sich Sätze wie (4), bei denen eine Reduktion auf einfache Sätze nach einer Regel in der Art der von Chomsky (1957) gegebenen nicht möglich ist.

(4) hans und maria lieben einander

Solche und ähnliche Beispiele führten Lakoff und Peters (1966) dazu, als Quelle koordinierter Einheiten neben der Transformierung mehrerer einfacher Sätze, genauer: der Transformierung einer Einheit, die mehrere koordinierte einfache Sätze enthält, auch einfache Sätze mit durch Ersetzungsregeln erzeugten komplexen Konstituenten (etwa NP + und + NP) zuzulassen. Treten in der zugrundeliegenden Kette derartige Koordinationen auf, so sprechen Lakoff und Peters von 'phrasal conjunction' und stellen diese der 'sentence conjunction' gegenüber, bei der die Konjunkte in der zugrundeliegenden Kette Sätze sind, die jedoch durch Transformationen auf eine oberflächliche Koordination von Phrasen abgebildet werden können.

Zum anderen wurde das Reduktionspostulat von Smith (1969) aufgegeben, um dem Ambiguitätskriterium bei Sätzen wie (5),

(5) hans und fritz fahren nach amerika

die als mehrdeutig angesehen wurden ('Hans und Fritz fahren zusammen/nicht-zusammen nach Amerika'), Rechnung zu tragen.

Damit war das Feld freigegeben, für alle möglichen und unmöglichen Versuche zur Erzeugung koordinierter Einheiten die Mittel 'phrasal conjunction' und 'sentence conjunction' als Regeln oder als Regelschemata jeweils ausschliesslich oder in Kombination mit wechselndem Schwergewicht zu verwenden. (Ein Überblick über die Zugehörigkeit der 
wichtigsten Publikationen zu den verschiedenen möglichen Positionen findet sich z.B. in Lang 1977: 284f, Anm. 1.)

Einige Problemfälle hatte bereits Gleitman, die die Reduktionsthese uneingeschränkt vertrat, behandelt. Sie schlug vor, Sätze wie (6) und (7) von den (8) bzw. (9) zugrundeliegenden Ketten abzuleiten:

(6) john and mary saw themselves

(7) john and mary saw each other

(8) john saw john mary say mary

(9) john saw mary mary saw john

Auf das Deutsche bezogen setzte zuerst Thümmel (1968) Gleitmans Bemühungen zur Rettung des uneingeschränkten Reduktionspostulats fort. In Bezug auf Gleitmans Vorschläge und ebenso hinsichtlich deren Anwendung durch Thümmel auf das Deutsche, scheint es bereits fragwürdig, wieso ein Zusammenhang, wie der zwischen Satzpaar (9) und Satz (7) ein syntaktischer sein sollte, der es erlaubt, die Form des Satzes (7) als Umformung von (9) zu beschreiben. Deutlicher zeigt sich die Verselbständigung des Apparats, bei der diejenigen Beziehungen als syntaktische reklamiert wurden, die die Syntaxmaschinerie herstellt, an Thümmels Behandlung von Sätzen wie (10) (Thümmel 1968: 409):

(10) die damen grüssten einander höflich

Da die Anzahl der Elemente einer durch ein Pluralnominal bezeichneten Menge unbestimmt ist, kann nach Thümmel (10) nicht in der für (7) vorgesehenen Art und Weise auf zugrundeliegende satzwertige Ketten reduziert werden. Andererseits erscheint es wünschenswert, Sätze mit einander immer gleichartig zu analysieren. Somit muss (11) von einer ähnlichen Kette wie (10) abgeleitet

(11) peter und felix schlagen einander

werden. Thümmels Lösungsandeutung sieht wie folgt aus. (10) liegt (12) zugrunde (mit ' $\Delta$ ' als 'dummy symbol'):

(12) die damen (jede dame die andere dame höflich grüsst) $\Delta$ 
In (12) soll der eingeklammerte Teil einen in den Matrixsatz 'die damen $\Delta$ ' eingebetteten Konstituentensatz darstellen. Analog wird (11) von der Kette (13) abgeleitet:

(13) peter und fritz (jeder den anderen schlägt) $\Delta$

Das Reduktionspostulat soll nun weiter gelten können, weil der Matrixsatz ' peter und fritz $\Delta^{\prime}$ ' in (13) wiederum auf eine Satzkoordination reduziert werden soll.

Mit einigem Aufwand an generativer Maschinerie liefert Thümmel Reduktionen koordinierter Einheiten auf Satzkoordination für eine Reihe weiterer Problemfälle, die hier nicht mehr im einzelnen besprochen werden können. Etwa wird erwogen (14) auf (15) zu beziehen:

(14) felix und peter sind brüder

(15) felix ist bruder von peter und peter ist bruder von felix

Selbst unter Voraussetzung von Thümmels Vorschlägen bleiben aber Sätze wie (16) irreduzibel:

(16) hans und maria sind ein ehepaar

Bemerkenswert an Thümmels Untersuchung ist, dass das Reduktionspostulat, das zunächst in erster Linie hinsichtlich der Vereinfachung der Grammatik gerechtfertigt worden war, nun selber als Grundlage für die Einführung von Regeln von beträchtlicher Komplexität herhalten muss. Unter Ausnutzung der Unklarheit des Verhältnisses von Syntax und Semantik in den Arbeiten Chomskys stützt sich Thümmel deshalb auf die Auffassung, dass das Reduktionspostulat in die generative Grammatik aufgrund semantischer Erwägungen aufgenommen worden sei. Nach der Konzeption semantischer Interpretation im zweiten TG-Modell sollten die Ausgangsketten der transformationellen Ableitung der Oberflächensätze 'Tiefenstrukturen' konstituieren, die die Bedeutungen der Sätze vollständig festlegen. Nun zeigte sich jedoch, dass gerade bei einer solchen Konzeption von Tiefenstruktur eine Reduktion komplexer Sätze auf Ketten, die Verknüpfungen einfacher Sätze in etwa entsprachen, für koordinierte Einheiten nicht möglich war, da die in Frage kommenden zugrundeliegenden Satzkoordinationen mit den von ihnen abgeleiteten Oberflächensätzen nicht semantisch äquivalent waren. Diese Ansicht wurde insbesondere von 
Wierzbicka prononciert vorgetragen (vgl. Wierzbicka 1972). Für das Deutsche legte Harweg (1970) eine Studie vor, in der gezeigt werden sollte, dass bei einem hinreichend anspruchsvollen Begriff von semantischer Äquivalenz eine 'Reduktionsanalyse' für koordinierte Einheiten nur in speziellen Ausnahmefällen erwägenswert sei. Wenngleich die Frage, für welche Fälle die Reduktionshypothese haltbar sei, umstritten blieb, ergab sich generell aus der Forderung, nach der die Transformationen, die die Tiefenstrukturen in Oberflächenstrukturen überführen, wegen der vollständigen Bedeutungsfestlegung in der Tiefenstruktur 'bedeutungserhaltend' sein sollten, ${ }^{6}$ dass die Frage einer syntaktischen Begründung der angenommenen Tiefenstrukturen als syntaktische Strukturen der Sätze der betrachteten Sprache immer mehr durch die semantischen Anforderungen ersetzt wurde.

Eine Schilderung der weiteren Entwicklungen und Richtungsaufspaltungen der generativen Grammatik würde an dieser Stelle den Rahmen sprengen. ${ }^{7}$ Verschiedenste der vom Beschreibungsmittel zum Beschreibungsgegenstand avancierten Tiefenstrukturen wurden auch für koordinierte Einheiten erwogen. Generell kann festgestellt werden, dass die zunehmende 'Abstraktheit' der Tiefenstrukturen gegenüber den Oberflächenstrukturen auch zunehmend die Erfüllung der Grundforderung an generative Grammatiken - die Spezifizierung der wohlgeformten Einheiten der zu beschreibenden Sprache - erschwerte. Ein Beispiel (vgl. Rivero 1970) ist die Annahme von 'Negationselementen' in der Tiefenstruktur, um derentwegen Wörter wie sin, span. 'ohne', grob gesprochen in no con, 'nicht mit', aufgelöst wurden. Die syntaktische Tatsache aber, dass Sätze gebildet werden können, die $\sin$ und no enthalten, deren 'Varianten' mit zwei Vorkommen von no jedoch nicht wohlgeformt sind, wurde durch die angenommenen Strukturen gerade verdeckt, so dass die Erzeugung z.B. von entsprechenden Sätzen mit zwei Vorkommen von no besonders verhindert werden musste.

Bei der Untersuchung der Mittel, mit denen die 'generative Kraft' der Transformationsgrammatiken wieder so beschränkt werden sollte, dass die Erzeugung nicht-wohlgeformter Oberflächenstrukturen verhindert

\footnotetext{
${ }^{8}$ Vgl. aber Partee $(1970,1971)$.

${ }^{7}$ Einen grossen Raum nahm auch die Diskussion um Koordinationsuniversalien ein, die durch Ross' (1970) Aufnahme der Greenbergschen Sprachtypologie (1966) ausgelöst wurde. Der ambitionierteste Beitrag war Harries (1973), der zuletzt von Carroll (1978) weitergeführt wurde. Der Gegensatz zwischen breitem empirischen Material und völlig spekulativen theoretischen Vorschlägen war hier besonders krass, vgl. Pullum (1974) und - aus nichtgenerativer Sicht - Walter (1976).
} 
werden konnte, wurden in einer Reihe von Dissertationen zu Ende der sechziger Jahre 'Constraints' vorgeschlagen, die etwa in dem Verbot von Transformationen mit gewissen Eigenschaften bestanden. Für koordinierte Einheiten relevante Constraints wurden insbesondere in Ross (1967) vorgeschlagen. Im Laufe dieser Untersuchungen wurde auch den Oberflächenstrukturen wiederum mehr Aufmerksamkeit geschenkt ${ }^{8}$ und die Auffassung von der Notwendigkeit von 'Beschränkungen über Oberflächenstrukturen' zuerst in Ross (1967), dann in den Dissertationen von Rivero und Perlmutter entwickelt, die ihren Niederschlag in Rivero (1970) und Perlmutter (1970) fanden. Nach diesen Arbeiten kann man die Erzeugung ungrammatischer Einheiten nicht immer durch die 'Blockierung' von Transformationen verhindern, die Chomsky (1965) angenommen hatte. Stattdessen "one must state a well-formedness condition that the output of the transformational component must meet" (Perlmutter 1970: 189). Wie Perlmutter selbst bemerkt (250), haben seine "Surface structure constraints' Ähnlichkeit mit Oberflächenbeschreibungen ausserhalb der generativen Schule. Die Oberflächenbeschränkungen liefern jedoch alleine nicht die Wohlgeformtheitsbedingungen für Oberflächenstrukturen, da sie solche Einheiten nicht ausschliessen brauchen, zu denen es gar keine Tiefenstrukturen gibt.

Die Diskussion der Oberflächen-Constraints hat Schachter in seinen Arbeiten aufgenommen und in Schachter (1977) auf das Thema Koordination angewendet. In dieser Arbeit versucht Schachter die Gleichartigkeitsforderung an Konjunkte durch eine 'Beschränkung über Oberflächenstrukturen' zu rekonstruieren, die er 'Coordinated Constituents Constraint (CCC)' nennt. Der CCC besagt, dass "coordinately conjoined constituents must be identical in syntactic category and semantic function" (1977: 102). Der erst Teil dieser Forderung war bereits in die oben zitierte Regel aus Chomsky (1957) eingeflossen. Sie muss nach Schachter u.a. deshalb für Oberflächenstrukturen und nicht für Tiefenstrukturen erhoben

${ }^{8}$ Die unterschiedlichen Gründe für das erstarkte Interesse an Oberflächenstrukturen innerhalb der generativen Grammatik hängen alle damit zusammen, dass die 'Tiefe' der Beschreibung den Zugriff auf wichtige Eigenschaftęn sprachlicher Einheiten versperrt. So ist Chomsky seit seinen ersten Hinweisen auf die Notwendigkeit, bei der semantischen Interpretation auf Eigenschaften der Oberflächenstrukturen Bezug zu nehmen (1965: 224, Anm. 9), über verschiedene Zwischenstufen zu der Auffassung gelangt, dass "it seems reasonable to postulate that only surface structures undergo semantic interpretation, though our 'surface structures' are no longer those of the standard theory" (1976: 96). Wegen der Möglichkeit "to carry over to surface structures certain properties of phrase markers that initiate derivations or that appear at an intermediate stage of derivation" (1976:117) sind es in der Tat keine Oberflächenstrukturen. 
werden, weil in der Tiefenstruktur Einheiten, die tatsächlich nicht koordinierbar sind, zu ein und derselben Kategorie gehören können und daher ihre Erzeugung nur auf der Ebene der Oberflächenstruktur blockiert werden kann, auf der die oberflächlichen Kategorien zur Verfügung stehen, mit anderen Worten, der Grund ist der angeführte, dass die für die syntaktische Beschreibung relevanten Informationen in den Tiefenstrukturen abhanden gekommen sind.

Als Beispiel für die Verletzung semantischer Restriktionen führt Schachter (17) an

(17) they made john an offer and an officer

und schreibt: "an offer is functioning as a direct object, an officer as an object complement" (92). Diese und andere Charakterisierungen "semantischer' Abweichung bei Schachter legen es jedoch nahe, dass die Bezugnahme auf semantische Funktionen durch die Bezugnahme auf syntaktische Relationen der Oberflächenstruktur ersetzt werden kann. Dass Schachter nicht so verfährt, hängt mit Jackendoffs zweifelhafter These (1972: 25) der "semantic insufficiency of grammatical relations" zusammen und führt mit dieser Version der These der inexakten Beziehung einmal mehr auf das ungeklärte Problem, wie Syntax und Semantik zusammenhängen.

Die gleiche Unklarheit hinsichtlich des Status der Forderung nach Kategorienidentität, die ich oben in Hinblick auf das erste Modell der TG festgestellt hatte, findet sich auch bei Schachter wieder. Schachter nimmt die Frage ausdrücklich auf (1977:89):

"In the majority of cases, constituents whose conjunction is grammatical would be assumed to belong to the same syntactic category on independent grounds, and constituents whose conjunction is ungrammatical would $[. .$.$] be assumed to belong to different categories; but$ there are apparent exceptions in both directions. I would suggest that such exceptions do not invalidate the generalization that coordinate conjunction requires category identity, but rather show that our assumptions about categorization may require some refinement."

Dies scheint in Übereinstimmung mit der von Schachter zuvor getroffenen Feststellung zu stehen (1977: 87; meine Hervorhebung, B.W.):

"The CCC, then, will require that coordinately conjoined constituents belong to the same syntactic category."

Er schliesst jedoch die zuvor zitierte Passage mit dem Satz:

"As suggested by Chomsky [...] the possibility of coordinate conjunction may perform a heuristic function in this regard." 
In der Praxis läuft dies auf die Postulierung der erwünschten Kategorien hinaus, so z.B. wenn für das Adverb quickly und die Präpositionalphrase with good appetite wegen festgestellter Koordinierbarkeit in einer entsprechenden Konstituentenstruktur jeweils zusätzlich ein Knoten AdvP (Adverbialphrase) angenommen wird.

Wenn die Wohlgeformtheit von Sätzen mit Hilfe des Apparats grammatischer Transformationen nicht zu spezifizieren ist, sondern Bedingungen angegeben werden müssen, die hinsichtlich der Oberflächenstrukturen der Sätze formuliert werden müssen, so liegt es natürlich nahe, auf die Transformationen, die sich als überflüssig erwiesen haben, zu verzichten. Tatsächlich wird dieser Schritt in der Arbeit von Hudson (1976a) ${ }^{9}$ gemacht. Hudson stellt drei Regeln zur Erzeugung der Strukturen koordinierter Einheiten auf und kommt zu dem Ergebnis: "All three rules apply to a level of surface structure" (1976a: 535) und er geht weiter und nimmt an (1976a: 561): "It might not be so difficult to generate all the structures resulting from these three rules without recourse to transformations at all."

Bei allen Änderungen, denen Transformationsgrammatiken unterzogen wurden, blieben die Grundschwierigkeiten, die sich mit dem ersten Modell gezeigt hatten, bestehen. An der Beschreibung der Koordination als einer wichtigen Teilaufgabe der Syntax bestätigt sich mithin die mehr allgemein gehaltene Einschätzung Hawkeys (1970: 193):

"While Syntactic Structures no longer occupies the sole preeminent position among the works of the transformationalists, the influence of the major theoretical notions presented therein has not lessened in the years since publication."

\section{Weitere Perspektiven}

Die Diskussion um die richtige Beschreibung der Koordination in der Transformationsgrammatik führte, wie der Überblick im vorhergehenden Abschnitt gezeigt haben sollte, zu

- der Erkenntnis der Wichtigkeit einer oberflächenstrukturellen Beschreibung

- der Erkenntnis der Wichtigkeit syntaktischer Relationen oder 'funktionaler Beziehungen'

sowie $\mathrm{zu}$

- der Erwägung, Transformationen als Beschreibungsmittel aufzugeben.

${ }^{\ominus}$ Vgl. auch die früheren Arbeiten von Hudson, insbesondere (1970), wo Hudson Diks Forschungen (s.u.) aufnimmt, und Hudson (1976b). 
Ausserdem litt die Aufstellung der Regeln unter der Unklarheit über die Empirizität der zu formalisierenden Feststellungen. Zusammen charakterisieren diese Gesichtspunkte eine Entwicklungstendenz, die in auffälligerweise bereits mit Diks Vorschlag (1968) für eine nicht-transformationelle generative Koordinationstheorie vorweggenommen wurde.

Dik hatte es unternommen, eine Phrasenstrukturgrammatik zu entwickeln, die den Verzicht auf Tiefenstrukturen durch die Einführung von Funktionsetiketten wie z.B. SUBJECT erlaubte. Obwohl Dik grossen Wert auf die Unterscheidung kategorialer und funktionaler (relationaler) Informationen legte, hielten seine Kritiker aus dem transformationellen Lager an der Ablehnung funktional gedeuteter Etiketten fest. Tatsächlich mögen Diks Rezensenten (vgl. Matthews 1969; Dougherty 1969) mit ihrer Vermutung nicht im Unrecht gewesen sein, dass der von Dik eingeführte Apparat seine eigenen Intentionen nicht richtig wiedergab.

Dik hatte die Verwendung von Funktionsetiketten der Definition syntaktischer Relationen auf der Grundlage syntaktischer Strukturen als Alternative gegenübergestellt. In der Transformationsgrammatik sollten die semantisch relevanten syntaktischen Relationen durch Angabe von 'Konfigurationen' in Tiefenstrukturen definiert werden. Würden die Tiefenstrukturen aufgegeben, so argumentierte Dik, müsste auch der Konfigurationsansatz fallen; dann aber stellte die Einführung von Funktionsetiketten eine Notwendigkeit dar (1968: 153):

\footnotetext{
"Within the full set of the linguistic structures of any one language, there does not seem to be a one-to-one correspondence between grammatical functions and configurations of categories. The reduction of the former to the latter is, consequently, excluded in principle.'
}

Mit der Infragestellung einer transformationellen Beschreibung der Koordination und den immer weiter verbreiteten Zweifeln am Wert von Transformationssyntaxen insgesamt, erhält die Überprüfung der von Dik gesetzten Alternative zwischen dem Konfigurationsansatz und dem Ansatz der Funktionsetiketten erneut grösste Bedeutung. Ich möchte diesen Aufsatz daher mit einigen Überlegungen abschliessen, die darauf hinweisen, dass zwischen beiden Ansätzen kein Wahlzwang besteht, sondern vielmehr der Konfigurationsansatz in einer nicht-transformationellen Beschreibung aufrechterhalten werden sollte.

Nach Dik ist es die Unmöglichkeit einer Eins-zu-eins-Zuordnung zwischen Oberflächenkonfigurationen und syntaktischen Relationen, die einen oberflächenorientierten Konfigurationsansatz verhindern müsste. Damit übernimmt Dik eines der Grundargumente, mit dem die Trans- 
formationalisten die 'Unzulänglichkeit' von Oberflächenstrukturen belegen wollen. Die 'Unexaktheit' der Beziehung zwischen Oberflächenstrukturen und Bedeutungen, die bei Chomsky den indirekten Weg der Bedeutungszuordnung über Kernsätze (oder Tiefenstrukturen) motivierte, bestand ja gerade darin, dass die relevanten Relationen nicht eineindeutig auf Oberflächenstrukturen zu beziehen waren.

Nun ist aber das Fehlen einer Eins-zu-eins-Zuordnung in gar keiner Weise ein Beweis für die Unmöglichkeit einer andersartigen und dennoch systematischen Zuordnung. Ebensowenig wie Homonymie und Synonymie eine prinzipielle Unexaktheit der Zuordnung von Wortformen und Wortbedeutungen nach sich ziehen, ebensowenig verhindern konfigurationell unterschiedliche Manifestationen einer syntaktischen Relation (oder umgekehrt die Mehrdeutigkeit einer Konfiguration hinsichtlich der bestehenden syntaktischen Relationen) eine systematische Beschreibung der Beziehung. Es ergibt sich allein, dass die Bestimmung syntaktischer Relationen mithilfe von Oberflächenkonfigurationen gegenüber den von Chomsky gegebenen von wesentlich grösserer Komplexität sein werden und im allgemeinen der Form nach eine Disjunktion von Bedingungen bilden werden. ${ }^{10}$

Darüberhinaus ist die Verbindung von Konfigurationsansatz und Oberflächengrammatik nicht nur nicht prinzipiell ausgeschlossen, sondern in Hinblick auf eine korrekte Verbindung von Syntax und Semantik höchst wünschenswert. Die Syntax sollte, wie oben angesprochen wurde, semantikfundierenden Charakter haben. Als Beispiel dieser Auffassung hatten wir Bloomfields Forderung herangezogen, Konstituentenanalysen in Hinblick auf die Satzsemantik zu bewerten. Gleichzeitig sollte der formale Aufbau syntaktischer Strukturen nicht in Frage gestellt werden, wie Bloomfield deutlich macht (1965: 168):

"The grammatical forms are no exception to the necessary principle - strictly speaking, we should call it an assumption - that a language can convey only such meanings as are attached to some formal feature."

Bei dieser Annahme wäre jede semantische Begründung syntaktischer Strukturen eine petitio principii. ${ }^{11}$ Entsprechendes gilt für die syntaktischen

\footnotetext{
${ }^{10}$ Freilich würde die Bestimmung der syntaktischen Relationen bei diesem Ansatz die Bezugnahme auf einzelsprachliche Fakten erfordern. Dies schliesst aber grundsätzliche Gemeinsamkeiten zwischen Relationen, die im einzelnen für verschiedene Sprachen unterschiedlich bestimmt sind, nicht aus. Vgl. Eisenberg (1976: 24ff).

${ }^{11} \mathrm{Zu}$ den Anforderungen an die Beziehung zwischen Syntax und Semantik vergleiche auch die klarsichtigen Formulierungen von Fries (1954) - einem Aufsatz, der eben zu der Zeit erschien, als Chomsky sein The Logical Structure of Linguistic Theory verfasste!
} 
Relationen, da diese einen entscheidenden Beitrag zum Aufbau von Satzbedeutungen liefern müssen. Wenn syntaktische Relationen nicht auf der Grundlage formaler Eigenschaften syntaktischer Einheiten bestimmt werden können, entbehren sie daher jeder Erklärungskraft. ${ }^{12}$

Die Durchführung des Programms einer semantikfundierenden Syntax ist seit einer Reihe von Jahren im Rahmen der Integrativen Sprachwissenschaft in Angriff genommen worden. Zur Konzeption dieses Ansatzes gehört es, dass die Satzsemantik wesentlich auf die semantische Interpretation syntaktischer Relationen gestützt wird, die ihrerseits auf die Eigenschaften formal bestimmter syntaktischer Strukturen gegründet werden. Die angenommenen syntaktischen Strukturen sind Oberflächenstrukturen in einem präzisierten Sinne, die angestrebten Syntaxen daher semantikfundierende Oberfächensyntaxen. Die bisherigen Ergebnisse lassen die Erwartung zu, dass auch die Anwendung auf Fragen der Koordination sich als fruchtbar erweisen wird. ${ }^{13}$

\section{Bibliographie}

Babitch, R.M., 1977. What has happened to Chomsky's concept of creativity. In: R.J. Di Pietro, E.L. Blansitt (Hrsg.), The Third LACUS Forum 1976, 337-341. Columbia: Hornbeam.

Bloomfield, L., 1965. Language. London: Allen and Unwin. $\left({ }^{1} 1933,{ }^{2} 1935\right.$ (revidiert), ${ }^{9} 1965$ ). Braun, S., 1967. Die Ausklammerung gemeinsamer Elemente in konjunktional verbundenen Wortgruppen im Deutschen. Beiträge zur Linguistik und Kommunikationsverarbeitung $11,72-88$.

Carroll, J.M., 1978. On coordination reduction. Lingua 46, 49-60.

Chomsky, N., 1955. The logical structure of linguistic theory. O.O. (vervielfältigt).

Chomsky, N., 1957. Syntactic structures. The Hague: Mouton.

Chomsky, N., 1965. Aspects of the theory of syntax. Cambridge: MIT Press.

Chomsky, N., 1966. Topics in the theory of generative grammar. The Hague: Mouton.

Chomsky, N., 1975. The logical structure of linguistic theory. New York/London: Plenur. Press.

${ }^{12}$ Die hier angesprochenen Punkte betreffen ebenso Diks neue Version einer Funktionalen Grammatik (1978), bei der Dik an seiner "basic idea" festhält, "namely the adoption of functional notions as primitives of grammatical theory" (V; meine Hervorhebung).

${ }^{13}$ In der Integrativen Sprachwissenschaft ist der Begriff "semantikfundierende Oberflächensyntax' präzisiert (vgl. Lieb 1977a als Zusammenfassung) und die Konzeption am Deutschen und Englischen erprobt worden. Abriss und Bibliographie des Ansatzes enthält (Lieb 1977b). Als Beispiel einer konfigurationellen Bestimmung einer syntaktischen Relation vgl. die Adjektivattributrelation (Eisenberg 1976: 33f). Zur Behandlung der Koordination werden in Wiese (1978) eine syntaktische Relation zwischen koordinierten Teileinheiten angenommen - die Koordinationsrelation - und deren Eigenschaften für Haupttypen der Koordination im Deutschen geklärt. 
Chomsky, N., 1976. Reflections on language. Glasgow: Fontana/Collins.

Chomsky, N., H. Parret, 1974. [Dialogue]. In : H. Parret (Hrsg.), Discussing language, 27-54.

The Hague: Mouton.

Dik, S.C., 1968. Coordination. Amsterdam: North-Holland.

Dik, S.C., 1978. Functional grammar. Amsterdam: North-Holland.

Dougherty, R., 1969. Rezension von Dik (1968). Language 45, 624-636.

Eisenberg, P., 1976. Oberflächenstruktur und logische Struktur. Untersuchungen zur

Syntax und Semantik des deutschen Prädikatadjektivs. Tübingen: Niemeyer.

Fries, Ch.C., 1954. Meaning and linguistic analysis. Language 30, 57-68.

Gleitman, L., 1965. Coordinating conjunctions in English. Language 41, 260-293.

Greenberg, J.H. (Hrsg.), 1966. Universals of language. Cambridge: MIT Press.

Harries, H., 1973. Coordination reduction. Working Papers on Language Universals 11, 139-209. Wiederabgedruckt in: J.H. Greenberg (Hrsg.), 1978, Universals of human language, Bd. 4: Syntax, 515-583. Stanford: Stanford Univ. Pr.

Harweg, R., 1970. Phrasale und-Koordination in der generativen Grammatik. Zeitschrift für Phonetik, Sprachwissenschaft und Kommunikationsforschung 23, 192-214.

Hawkey, R.L., 1970. A critique of certain basic theoretical notions in Chomsky's Syntactic Structures. Folia Linguistica 4, 193-209.

Helbig, G., 1971. Geschichte der neueren Sprachwissenschaft. München: Hueber.

Hockett, Ch.F., 1948. A note on 'Structure'. International Journal of American Linguistics 14, 269-271.

Hudson, R.A., 1970. On clauses containing conjoined and plural noun-phrases in English. Lingua 24, 205-253.

Hudson, R.A., 1976a. Conjunction-reduction, gapping, and right-node raising. Language $52,535-562$.

Hudson, R.A., 1976b. Arguments for a non-transformational grammar. Chicago/London: Univ. of Chicago Press.

Jackendoff, R.S., 1972. Semantic interpretation in generative grammar. Cambridge/London: MIT Press.

Katz, J.J., J.A. Fodor, 1963. The structure of a semantic theory. Language 39, 170-210.

Lakoff, G., St. Peters, 1966. Phrasal conjunction and symmetric predicates. Cambridge: Harvard Univ. Computation Laboratory.

Lang, E., 1977. Semantik der koordinativen Verknüpfung. Berlin: Akademie-Verlag.

Lieb, H., 1974. Grammars as theories: The case for axiomatic grammar (Part I). Theoretical Linguistics 1, 39-115.

Lieb, H., 1977a. Surface syntax as a basis for semantics. In: R.J. Di Pietro, E.L. Blansitt (Hrsg.), The Third LACUS Forum. Columbia: Hornbeam.

Lieb, H., 1977b. Outline of integrational linguistics. Preliminary Version. Berlin: Freie Universität FB 16 (= Linguistische Arbeiten und Berichte Berlin (West) Nr. 9).

Lyons, J., 1968. Introduction to theoretical linguistics. Cambridge: Cambridge Univ. Pr.

Matthews, P.H., 1969. Review article [Rezension von Dik (1968)]. Lingua 23, 349-371.

Partee, B.H., 1970. Negation, conjunction and quantifiers: syntax vs. semantics. Foundations of Language 6, 153-165.

Partee, B.H., 1971. On the requirement that transformations preserve meaning. In: Ch.J. Fillmore, D.T. Langendoen (Hrsg.), Studies in linguistic semantics, 1-22. New York: Holt.

Paul, H., 1959. Deutsche Grammatik, Bd. 3: Teil IV: Syntax. Halle: Niemeyer (5. Auf.).

Perlmutter, D.M., 1970. Surface structure constraints in syntax. Linguistic Inquiry 1, $187-255$.

Pullum, G.K., 1974. A note on coordination reduction and word order in Hindi. Working Papers on Language Universals 14, 95-101. 
Rivero, M.-L., 1970. A surface structure constraint on negation in Spanish. Language 46, 640-666.

Ross, J.R., 1967. Constraints on variables in syntax. Cambridge: MIT (Diss.) (vervielfältigt).

Ross, J.R., 1970. Gapping and the order of constituents. In: M. Bierwisch, K.E. Heidolph (Hrsg.), Progress in linguistics, 249-259. The Hague: Mouton.

Schachter, P., 1977. Constraints on coordination. Language 53, 86-103.

Smith, C.S., 1969. Ambiguous sentences with and. In: D.A. Reibel, S.A. Schane (Hrsg.), Modern studies in English, 75-79. Englewood Cliffs: Prentice Hall.

Thümmel, W., 1968. Deutsche und-Koordination und die rekursive Kapazität der Transformationsgrammatik. Lingua 20, 381-414.

Thümmel, W., 1970. Formale Schwierigkeiten bei der Beschreibung von Satzverknüpfungen mit Hilfe von Konstituentenstrukturregeln. Muttersprache 80, 145-155.

Walter, W., 1976. Gapping, Wortstellung und Direktionalitätshypothese. Arbeitspapier Nr. 30. Köln : Institut für Sprachwissenschaft, Universität Köln.

Watt, W.C., 1974. Mentalism in linguistics, II. Glossa 8, 3-40.

Weydt, H., 1976. Noam Chomskys Werk. Tübingen: TBL Verlag.

Wierzbicka, A., 1972. And and plurality (against 'Conjunction Reduction'). In: A. Wierzbicka, Semantic primitives, 166-190. Frankfurt/M.: Athenäum.

Wiese, B., 1978. Die Koordinationsrelation im Deutschen. Berlin: Freie Universität FB 16 (MA-Arbeit). 